\title{
Discrete-continual mathematical models in solving vibration protection problems of building structures
}

\author{
Vladimir Sobolev ${ }^{1, *}$ \\ ${ }^{1}$ Irkutsk National Research Technical University, 664074, Lermontova str., 83, Irkutsk, Russia
}

\begin{abstract}
The paper outlines the method of forming mathematical models for the dynamic interaction of the vibroactive technological equipment with load-bearing structures of industrial structures on the basis of discretecontinual approximations previously developed by the author. Ways of implementing methods in the formation of vibration protection systems of structures are provided in the paper. The features of systems' functioning and their sensitivity to fluctuating vibration frequency processes are also described.
\end{abstract}

The prevalence of vibration technologies makes it necessary to pay special attention to the safety of building structures and workplaces [1-3]. These issues are especially acute in enrichment and other industries, the specific technology of which requires placing the vibroactive equipment on the upper floors, which excludes the possibility of using technological foundations and leads to direct transfer of dynamic loads to the bearing structures of the floors. The vibration system of such structures (which is a complex system of beams that is irregular in location, geometric parameters and junctions) is most intensively manifested by vertical components of the oscillations caused by operating modes of technological equipment [4-6]. Examples include mining and processing industries, energy, coal, wood processing industries, ship transport systems, etc., where screens, fans, electric motors, generators, compressors, and other vibrating equipment are installed. In cases where the vibration load is of a concentrated nature and the number of "points" of the load is small, it is advisable to use special devices to eliminate or reduce the intensity of vibration at the "points" of impact. Such a problem arises in the protection of a deformable base from the vibrational effects of a technological object, in particular, a screen. In this case, it is advisable to use a continuum-discrete model (CDM, which allows the implementation of the methods for determining the coordinates of the nodal points of oscillations (NPO) of the system's deformable part, with subsequent support at these points. This method of vibration isolation [7], which allows obtaining fairly simple realizations using elastic properties bending elements, is illustrated in Fig. 1. Vibroactive object in the form of a solid body with a mass $M$ with springs with rigidity $\tilde{N}$ of the supports on the elastic bend beam with stiffness $E J$ and distributed mass p, having a concentrated mass $m$ being fixed in the span between the springs support points.

\footnotetext{
* Corresponding author: vladsobol@yandex.ru
} 
The solution of individual problems of constructive formation of zero reactions at given points of support of a beam is reviewed in a number of papers [8-10].

The following techniques that underlie the method of vibration isolation are based on the solution of the inverse problem. They make possible to obtain a general approach and clearly illustrate the effects studied when analyzing the forced vibrational forms of continual elements.

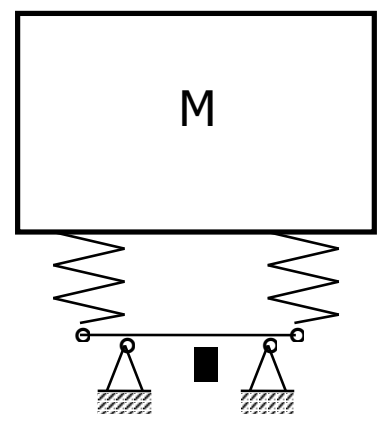

Fig. 1. Scheme of vibration isolation of a solid.

The simplest version of the problem of determining the coordinates of vibrational nodes occurs when the reactive mass is concentrated in the middle of the span of a weightless beam. Consider the design scheme (Figure 1.), differing in that the beam is not supported on the stationary supports, but oscillates under a harmonic action that moves the spring support points according to the law $\sin \omega t$.

Using the symmetry of the design scheme (Fig. 2), with respect to the vertical axis passing through the fixing point of the reactive mass, we write the equation of dynamic equilibrium

$$
-\frac{3 E I}{l^{3}}+\left(\frac{3 E I}{l^{3}}-m \omega^{2}\right) y_{1}=0
$$

of displacing the reactive mass during the harmonic movements $\sin \omega t$ of the reference point, determine the magnitude of the bending moment $M(X)$ in the span of the beam (taking into account the zero section rotation at the point of attachment of the mass provided by the symmetry of the design scheme).

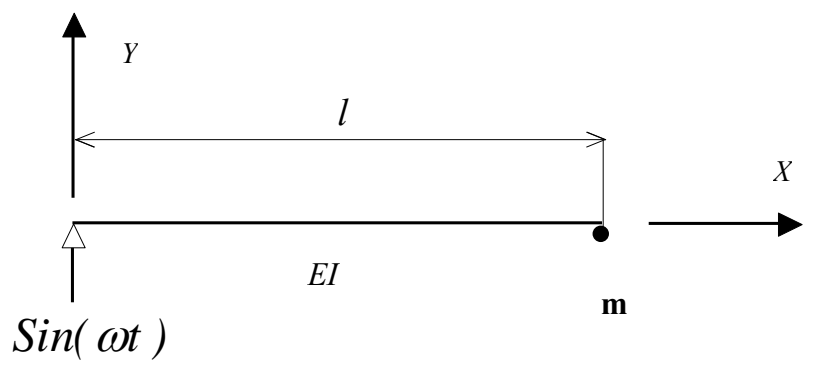

Fig. 2. Symmetrical design scheme of oscillations of the unpertured beam with harmonic displacements of the end nodes.

$$
M(x)=\frac{3 E I}{l^{3}}\left(\frac{3 E I}{3 E I-l^{3} m \omega^{2}}-1\right) x=0 .
$$


Integrating $M(x)$ and dividing the resulting expression by $E J$, we obtain the amplitude of rotations of the beam sections from the current coordinate $X$.

$$
\frac{d y(x)}{d x}=\int \frac{3}{l^{3}}\left(1-\frac{3 E J}{3 E J-l^{3} m \omega^{2}}\right) x d x+c_{1}
$$

Having determined $\tilde{n}_{1}$ from the conditions $\left.\frac{d y(x)}{d x}\right|_{x=l}=0$ and integrating again, we obtain

$$
y(x)=\frac{3}{l^{3}}\left(1-\frac{3 E J}{3 E J-l^{3} m \omega^{2}}\right)\left(\frac{x^{2}}{2}-\frac{l^{2}}{2}\right) d x+c_{2} .
$$

Having determined $c_{2}$ from the conditions $y(0)=1$, we have

$$
y(x)=\frac{3}{l^{3}}\left(1-\frac{3 E J}{3 E J-l^{3} m \omega^{2}}\right)\left(\frac{x^{3}}{6}-\frac{x l^{2}}{2}\right)+1 .
$$

The obtained function is a function of the vibration amplitudes (the shape of the forced oscillations) of the beam for various values of the design parameters and an arbitrary frequency of the action $\omega$. Transformation of the vibrational shape with a change in the frequency of the action is shown $\omega$ in Fig.3.

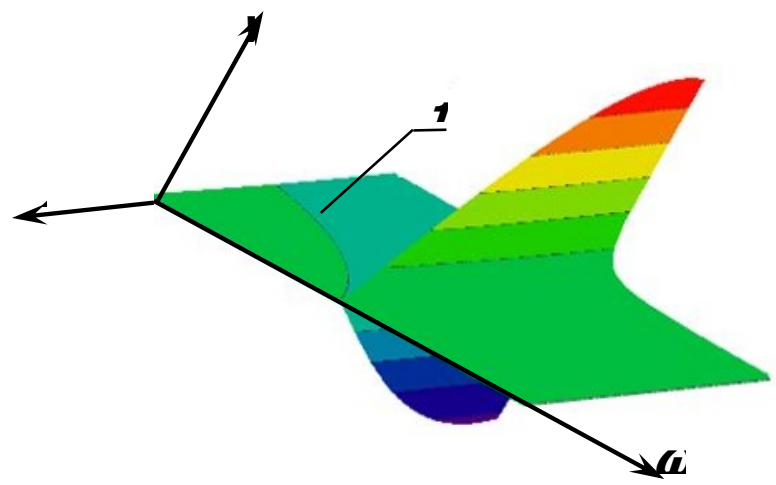

Fig. 3. Transformation of the vibrational form of a weightless beam with a discrete mass with a change in the frequency of the action $\omega$. (Infinite values at the resonance are burdened by the discreteness of plotting). " 1 " is the nodal line.

Consideration of a symmetric system without taking into account the mass of the beam, the displacement of which is described by expression (5), has shown that the shape of the beam oscillations smoothly changes as $\omega$.

In this case, a single-phase movement of all beam points coincides with the harmonic effect in phase. For arbitrarily small values of the frequency $\omega$, nodal points are at an infinite distance from the point of impact. As you increase $\omega$, a monotonic approach of 
the point of the vibrational node to the point of impact occurs. Thus, the nodal points in the span of the beam, oscillating in the pre-resonance regime, are absent. To design a vibration isolation system that satisfies the conditions $\omega<\sqrt{\frac{3 E J}{l^{3} m}}$, it is necessary to ensure the support of the beam beyond the span between the supports of the screen.

If the value $\omega=\gamma$ corresponding to the natural frequency of the beam oscillations, the function of the shape of the forced oscillations undergoes a sharp change, characterized by the discontinuity of the function's $(y(x, \omega))$ amplitudes of the movements of each point $x$ of the beam axis in frequency $\omega$.

In this case, all the points of flight begin to oscillate in antiphase with respect to the harmonic effect. An exception is the point $\mathrm{x}=0$, in which an oscillating knot arises. The node monotonically moves to the point $\mathrm{x}=1$, as the values of $\omega$ increase and asymptotically reaches this point with $\omega \rightarrow \infty$. The nodal line in Figure 4 is depicted as a border of light shades reflecting the displacement of the vibrational phases in the resonance part of the volumetric plot.

Thus, with sufficient magnification $\omega$, a tendency appears to merge the two vibrational nodes into one at the attachment point of the reactive mass at $x=l$. Such an option is certainly unsuitable because of the static instability of the system. Avoid this by possibly increasing the ratio $\frac{E J}{m}$, which is accompanied by the removal of the vibrating assembly from the attachment point of the reactive mass.

Equations for determining the coordinates of the vibrational nodes are obtained by equating the function (5) to zero.

Very simple and obvious dependencies are obtained when using relative variables. Having made simplifications and passing to the relative coordinates in fractions of the value of $l$, we have:

$$
q^{3}-3 q+2-2 g^{2}=0
$$

where $q=\frac{x}{l}$ is relative coordinate of the vibrating unit, $g=\frac{\gamma}{\omega}$.

Thus, the coordinate of the vibrational node is uniquely determined by the ratio of frequencies and vice versa. For the resonant mode of oscillation $0<g \leq 1$.

The graph of the dependence (6), which plays an important role in the design of a CB, is shown in Fig. 4. As can be seen, it is a curve connecting the opposite angles of the unit square and slightly removed from its diagonal.

The equation (6) has a unique real solution that determines the coordinate of the node point for given design parameters $m, E J, l$, and the frequency of the impact $\omega$. Equality is also decisive for the solution of the inverse problem: finding the constructive quantities $\mathrm{m}$ or $E J$ and forming the vibrational node at a point with a given coordinate $x$.

For an asymmetric problem, when the center of mass and stiffness is displaced relative to the middle of the beam span (such conditions are typical for Schenk type screens), the equations for determining the coordinates of nodal points are derived similarly. In this case, the static indeterminability of the beam relative to the movements of the reactive mass is previously disclosed and the rotation of the cross-section of the beam at the point of its (mass). 


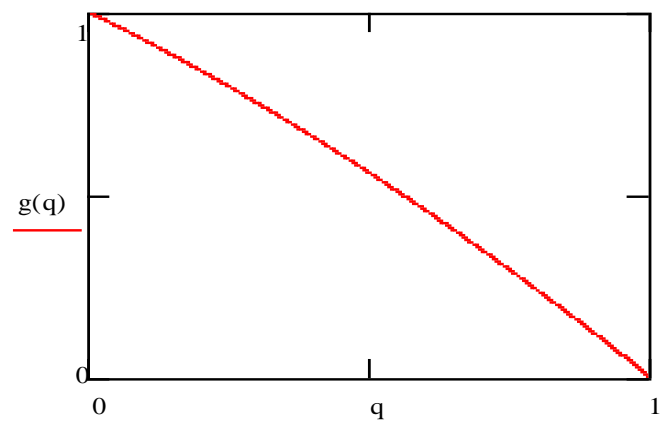

Fig. 4. Relative expression of frequencies $g$, forming a nodal point with coordinate $q$ in forced oscillations of bending.

To study the joint effect of the concentrated reactive and distributed beam mass on the dynamic properties of the vibration isolation system, the technique described in the work [7].

The obtained equation of dynamic equilibrium, compiled for the amplitude value of the displacement of the reactive mass under the action of harmonic displacement $\delta \sin \omega t$, has the form

$$
\begin{aligned}
& -\delta u^{3} \frac{E J}{l^{3}} \frac{\operatorname{ch} u+\cos u}{\operatorname{ch} u \sin u-\operatorname{sh} u \cos u}+ \\
& y_{2}\left(u^{3} \frac{E J}{l^{3}} \frac{2 \operatorname{ch} u \cos u}{\operatorname{ch} u \sin u-\operatorname{sh} u \cos u}-m \omega^{2}\right)=0
\end{aligned}
$$

where $u=l \cdot \sqrt[4]{\rho \omega^{2} / E J}, \rho$ is the mass of the running meter of the beam, $m$ is the value of the reactive concentrated mass.

Since the coordinates of the node points of oscillations of a system with zero dissipation are independent of the amplitude of the oscillations, the amplitude of the action $\delta$ can be set equal to one.

Having determined the amplitude $y_{2}$ of displacements from (7), the masses $\mathrm{m}$ and the coefficients $d_{1}-d_{4}$ of the linear combination of particular solutions in the general solution of the beam oscillation equation

$$
Y(x)=d_{1} \cos \gamma x+d_{2} \sin \gamma x+d_{3} \operatorname{ch} \gamma x+d_{4} \operatorname{sh} \gamma x,
$$

we obtain the function of the amplitudes of transverse displacements of the axis of the beam (Figure 5).

Here, $Y=\left(1, y_{2}, 0,0\right)$ is the vector of amplitude values of displacements along the direction of the bonds (vector of boundary conditions), $D=\left(d_{1}, d_{2}, d_{3}, d_{4}\right)^{\mathrm{T}}, A$, is the resolving matrix of the form

$$
A=\left[\begin{array}{c}
H(0) \\
H(l) \\
\left.H_{x}(x)\right|_{x=l} \\
\left.H_{x x}(x)\right|_{x=0}
\end{array}\right],
$$


defined by the beam design; $H=(\cos (\gamma x), \sin (\gamma x), \operatorname{ch}(\gamma x), \operatorname{sh}(\gamma x))$ is the vector-function of basis solutions of the equation of beam's oscillations, $\gamma=\sqrt[4]{m \omega^{2} / E J}$. The intersection of the beam's deformed axis with the $x$ axis at a $\omega$ fixed value determines the coordinates of the node points from solving the equation

$$
Y(x)=0 .
$$

The sequence of nodal points during the change $\omega$ forms a nodal line [11].

Function analysis

$$
Y(x)=d_{1} \cos \gamma x+d_{2} \sin \gamma x+d_{3} \operatorname{ch} \gamma x+d_{4} \operatorname{sh} \gamma x
$$

shows that the use of CDM in contrast to the discrete model leads to the formation of new bifurcation "branching" $[12,13]$ nodal lines as the frequency of exposure increases $\omega$. The branching process is determined by the complication of the forms of forced oscillations, being subject to changes passing through the resonance points as the $\omega$ changes.

The formation of nodal lines for an infinite-dimensional beam with a concentrated mass is shown in Fig. 5.

A sharp increase in the amplitudes of the oscillations corresponds to resonant values $\omega$. The step of the grid for plotting the graph sets off the peak values of the resonant amplitudes and in the figure they are represented by finite quantities. In the area of the graph to the first resonance frequency, there are no nodal points in the span of the beam. At a value equal to the frequency of the first resonance, a nodal point appears, coinciding with the point of support of the screen to the beam. Since the left side of the symmetric model is considered, there are two such points on the full model. The intersection of any resonance point leads to a sharp change in the vibrational form with bifurcation branching of nodal lines that are continuous in the interresonance zone $\omega$.

When using beams with a negligibly small distributed mass parameter $\rho$ (a weightless beam), there is no second resonance. Since the model with distributed parameters, being infinite-dimensional [14], has an unrestricted spectrum, changes in vibrational forms, branching of nodal lines occurs an infinite number of times. In this case, an increase in the number of vibrational nodes is due to the complication of the shape of the oscillations.

It is known that a real oscillatory system can be constructed with sufficient certainty only when several first frequencies are used that satisfy the accepted assumptions of elastic oscillations of transverse bending.

Obviously, the most preferred for the construction of a vibration isolation system is the resonant region of the first natural frequency, which causes the existence of two vibrational nodes (for the complete model). The coordinate of the first node point (the left side of the model) is determined by the real root of equation (6). 


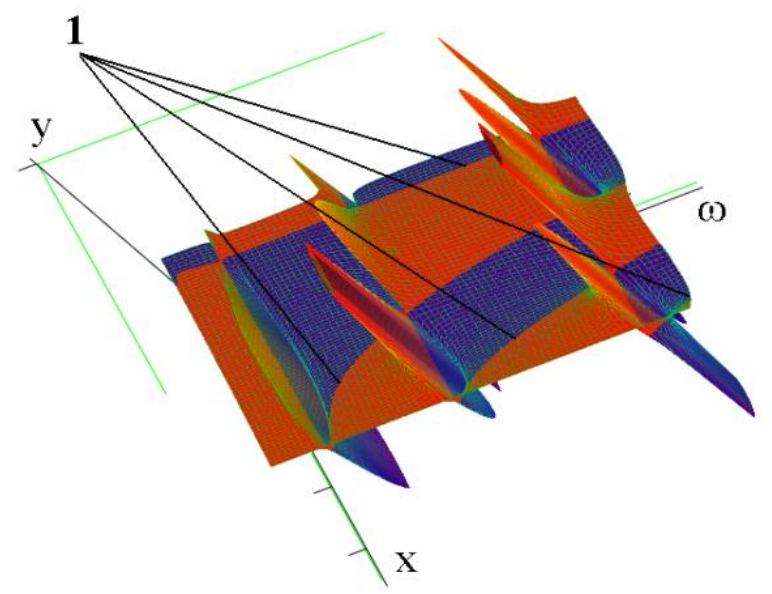

Fig. 5. Transformations of the forced vibrational forms of the beam axis with a change in the frequency of the impact $\omega: 1$ stands for nodal oscillation lines.

The subsequent leaning at these points (beams on the base) allows to obtain zero dynamic reactions in the beam supports. Thus, the proposed system allows to obtain zero dynamic responses to underlying building structures, which essentially relates it to the section of dynamic blanking systems $[15,16]$. A feature of such a system of vibration suppression is the fact that an element of load-bearing structures can be used as an element of a dynamic system, an inertial element with distributed inertial parameters. It should be noted that the study of the properties of vibration isolation of such systems based on nodal lines is practically absent in the literature and further study of such properties is, in our opinion, quite relevant.

\section{References}

1. B. G. Koreneva, I. M. Rabinovich, Dynamic calculation of structures for special effects (Stroyizdat, Moscow, 1981)

2. Yu. V. Flavitsky, Protection against noise and vibration at coal industry enterprises (Nedra, Moscow, 1990)

3. V. A. Ivovich, V. Ya Onishchenko, Protection from Vibration in Mechanical Engineering (Mechanical Engineering, Moscow, 1990)

4. Instruction on the calculation of load-bearing structures of industrial buildings and structures for dynamic loads (Stroyizdat, Moscow, 1970)

5. Instruction on measures to combat the vibrational effects of technological equipment in the design of buildings and structures of non-metallic building materials (Stroyizdat, Moscow, 1966)

6. Instructions for the determination of dynamic loads from machines installed on ceilings of industrial buildings (Stroyizdat, Moscow, 1966)

7. V. I. Sobolev, Discrete-continual dynamic systems and vibration isolation of industrial screens (Izdat. IrSTU, Irkutsk, 2002)

8. A. A Zhivotovsky, V. D. Afanasyev, Protection from vibrations and noise at mining enterprises (Nedra, Moscow, 1982)

9. Yu. V. Flavitsky, Protection against noise and vibration at coal industry enterprises 
(Nedra, Moscow, 1990)

10. V. V. Vasilevsky, IS Doronin, A. N. Shcherbakov, Vestnink VNIIZhT, 2, 36-38 (1984)

11. V. I. Sobolev, Bulletin of IrSTU, 2 (14), 89-92 (2003)

12. V. V. Amelkin, Differential equations in applications (Nauka, Nauka, Moscow, 1987)

13. V. I. Arnol'd, The theory ofcCatastrophes (Nauka, Chief Editorial Staff of Physical Literature, Moscow, 1990)

14. V. Koloushek, Dynamics of building structures (Publishing house of literature on construction, Moscow, 1965)

15. V. S. Glazyrin, Construction Mechanics and Design of Structures, 3, 43-47 (1971)

16. S. V. Eliseev, G. P. Nerubenko, Dynamic vibration dampers (Nauka, Novosibirsk, 1982) 